\title{
CONTRADICCIONES NORMATIVAS Y JERARQUÍA DE NORMAS
}

1 problema lógico de las contradicciones entre normas ha sido presentado y tratado de diferentes maneras por los lógicos, intentando por una parte dar cuenta de los problemas lógicos que deben enfrentar los juristas, y por otra parte proporcionar alguna solución.

Los trabajos hechos acerca de contradicciones normativas suponen en primer lugar la determinación de un concepto de «contradicción normativa» que permita identificar el problema, y en segundo lugar una idea clara de la relación entre la lógica y el derecho necesaria para proponer y evaluar cualquier propuesta de solución. En este artículo sostendré que el problema relevante es el de las contradicciones ${ }^{1}$ entre consecuentes de normas condicionales. Analizaré la propuesta de solución dada por Carlos Alchourrón en términos de lo que él llamó «condicionales derrotables»» ${ }^{2}$, propuesta que creo adecuada para ciertos fines. Creo, sin embargo, que resuelve más problemas de los que permiten los fundamentos que justifican su uso para la representación lógica del derecho. Esto es así por cuanto la formalización en términos de condicionales derrotables elimina las contradicciones del sistema y, de este modo, elimina el problema jurídico, en lugar de dar cuenta de él para facilitar su tratamiento. El problema lógico de la contradicción, en cambio, no ha sido adecuadamente identificado.

${ }^{1}$ La idea de que la forma más adecuada de representar las normas jurídicas supone el uso de un condicional (de algún tipo) es frecuente. Véase por ejemplo Alchourrón y Bulygin, Introducción a la metodología de las ciencias jurídicas y sociales, Astrea, 1975; Alchourrón, C., «Condicionalidad y la representación de las normas jurídicas», en Alchourrón, C. y Bulygin, E., Análisis lógico y Derecho, C.E.I., 1991.

${ }^{2}$ Alchourrón, C., «Condicionalidad y la representación de las normas jurídicas» (en Análisis lógico Derecho, ob. cit. nota 1). 
Diferenciaré los problemas lógicos de los sistemas jurídicos, que son los que preocupan a los juristas, de los problemas de la lógica aplicable a los sistemas jurídicos. Creo que los lógicos se avocaron a resolver el segundo intentando a la vez resolver el primero, lo que llevó a un tratamiento incorrecto de ambos.

Concluiré que se requiere un cambio en la lógica a fin de que sea apta para el análisis del derecho, y que la propuesta de C. Alchourrón basada en una lógica de condicionales derrotables, no es adecuada.

Sin una modificación de la lógica standard ${ }^{3}$ el problema jurídico de las contradicciones normativas no puede siquiera ser correctamente planteado.

\section{El problema de los juristas}

Una dificultad con la que frecuentemente se encuentran los juristas consiste en el hallazgo de normas contradictorias. El problema práctico consiste en que, ante ciertas condiciones de hecho, encuentran en el sistema varias normas aplicables que disponen soluciones inconsistentes. Intuitivamente podemos definir tal inconsistencia como la imposibilidad de que ambas normas sean obedecidas.

Veamos un caso hipotético ${ }^{4}$. Supongamos que están vigentes las siguientes normas:

a) Obligatorio detenerse ante los semáforos en rojo.

b) Prohibido detenerse en zona militar.

En el caso de que se emplazara un semáforo en zona militar, un jurista informado de este hecho reconocería seguramente un problema en el sistema de normas. Afirmaría que las dos citadas antes son inconsistentes, preguntándose en consecuencia cuál resulta aplicable.

Las alternativas que los juristas contemplan para casos similares son tres: 1.a) considerar aplicable la primera norma; 2.a) considerar aplicable la segunda ó 3.a) considerar inaplicables ambas.

${ }^{3}$ Considero lógica standard a la lógica proposicional y cuantificacional, con principio de no contradicción y de tercero excluido y el concepto tradicional de derivabilidad según la cual se requiere únicamente que la conclusión sea necesaria respecto de las premisas.

Como lógica deóntica standard (L. D.) tomo al sistema presentado por C. Alchourrón en «Lógica de normas y lógica de proposiciones normativas», en Alchourrón, C. y E. Bulygin, ob. cit. nota 1 .

${ }^{4}$ Citado por J. Rodríguezen «Contradicciones normativas: Jaque a la concepción deductivista de los sistemas jurídicos», en Doxa, 17-18. 
El fundamento para pronunciarse por cualquiera de las tres opciones usualmente sigue una de estas dos vías:

A) Se afirma que el derecho en realidad no es inconsistente sino que, para este caso, dispone la solución x (donde x consiste en la norma a), o bien en la norma b), o simplemente en la ausencia de solución normativa -el derecho, en realidad, se sostiene, no dice nada acerca de la conducta de detenerse ante semáforos en rojo en zonas militares, no contempla esta situación-).

B) El derecho es contradictorio, de modo que dispone tanto que es obligatorio detenerse como no detenerse en el caso planteado. Puede aplicarse una de las dos normas, o ninguna de ellas. La respuesta no puede obtenerse de un análisis del sistema jurídico.

Como se ve, el problema de las contradicciones normativas desde el punto de vista de los juristas puede consistir:

A) En identificar la solución jurídica correcta detrás de una aparente contradicción entre normas. Caso en el cual la llamada «contradicción normativa» no sería una contradicción en el sistema, sino una dificultad para interpretar correctamente ciertos textos o enunciados.

B) En identificar contradicciones entre normas, sin que la solución a los problemas que esto origine pueda darse «desde el derecho».

Quizás esta situación puede aclararse diciendo que, desde el punto de vista de un jurista, cuando surge el problema de una inconsistencia normativa sucede que, o bien no existe tal inconsistencia (una vez interpretado correctamente el derecho), o bien existe, pero no tiene solución (dada por el derecho).

El tratamiento que hace de este problema la ciencia jurídica (los juristas, y no los filósofos del derecho) consiste, o bien en establecer métodos adecuados para determinar el derecho existente (no contradictorio), o bien en identificar meramente las contradicciones o, a lo sumo, proponer cursos de acción para los casos en que el derecho, siendo inconsistente, no da respuestas.

El razonamiento del juez merece una aclaración especial. Éste, además de identificar el derecho debe aplicarlo, de modo que enfrenta el problema de que, ante la aparición de una contradicción debe tomar una decisión práctica, la cual supone él que debe estar totalmente determinada por el derecho. Así, aún cuando los criterios que normalmente use para el reconocimiento de normas jurídicas no le permitan resolver la contradicción, todavía sostendrá que el derecho realmente no es contradictorio, porque sí así fuera habría una indeterminación en el derecho (varias normas aplicables, entre las cuales debe seleccionarse porque, por razones lógicas, no pueden aplicarse simultáneamente) y éste no podría determinar su decisión. Por lo general el juez modifica sutilmente el criterio original de reconoci- 
miento del derecho que usó en el momento de identificar la contradicción, para adoptar ad hoc uno nuevo que la disuelva. Es una forma de modificar el derecho mismo para ese caso particular de modo que resulte consistente.

Esta manera de proceder muestra que el criterio común de reconocimiento de normas jurídicas (previo a la modificación ad hoc que hace el juez para evitar la inconsistencia) permite contradicciones, y ésta es la idea intuitiva que intento rescatar cuando afirmo, como haré en este trabajo, que la existencia de contradicciones en el derecho es una de las intuiciones jurídicas básicas.

En suma los juristas, o bien afirman que el derecho no es contradictorio, caso en que no existiría el problema a que nos referimos; o bien admiten que el problema existe, el derecho es contradictorio y dispone soluciones que no pueden aplicarse simultáneamente. Si se admiten contradicciones se dirá que las soluciones inconsistentes son jurídicamente admisibles, pero la selección no puede fundarse en el derecho.

Éste, creo, es todo el problema.

La lógica, en la medida en que es aplicable al análisis del derecho, debe ser capaz de dar cuenta de esta situación.

\section{El problema de los lógicos}

Una consecuencia formal de las contradicciones lógicas es, como se sabe, que ellas implican cualquier fórmula. Si en un sistema jurídico hubiera una contradicción, entonces en él sería derivable cualquier consecuencia; toda conducta estaría a la vez permitida y prohibida y el sistema completo sería inaplicable ${ }^{5}$.

Si un jurista aplicara la lógica deóntica standard al análisis de un sistema jurídico contradictorio llegaría a la conclusión absurda de que el sistema en su totalidad es inaplicable. Luego, se afirma, los juristas no hacen uso (no deben hacerlo) de la lógica deductiva formal en su actividad teórica (ni práctica).

El argumento es propuesto para demostrar que la lógica no puede ser aplicada al análisis del derecho. Quienes sostienen la utilidad de la lógica para el análisis del derecho ${ }^{6}$ intentaron demostrar 1) que la lógica no sólo puede aplicarse, sino que es necesaria para dar cuenta

${ }^{5}$ Esta forma de plantear el problema está presentada con claridad en Jorge Rodríguez, ob. cit. nota 4. Rodríguez reconstruye el argumento a partir de trabajos de autores como Lars Lindahl y Manuel Atienza.

${ }^{6}$ Ver, por ejemplo, Alchourrón, C. y Bulygin, E., Análisis lógico y Derecho, Introducción a la Metodología de las Ciencias Jurídicas y Sociales (obras cit. en nota 2); Lindahl, L., Position and Change, a Study on Law and Logic, D. Reidel Publishing Company, 1977. 
de los problemas que se plantean a los teóricos del derecho (el problema tradicional de las contradicciones normativas); y 2) que el análisis lógico de las contradicciones normativas es posible porque la aplicación de la lógica a sistemas jurídicos contradictorios no tiene los efectos que el argumento le atribuye.

\section{Un problema limitado}

Sostuve en otra parte ${ }^{7}$ que, aún concediendo que en un sistema normativo inconsistente cualquier solución es deducible, el problema tiene un alcance mucho más limitado que el que suponen quienes presentan el argumento, porque los juristas de hecho no suponen que haya conexiones lógicas entre todas las normas de un ordenamiento jurídico, sino que analizan conjuntos limitados de normas que tienen en común el referirse a un caso (genérico) determinado, y el atribuir un carácter deóntico a ciertas conductas (genéricas). De este modo, en el sentido lógico de «sistema» los juristas no suponen que el orden jurídico en su totalidad sea tal, sino más bien que el orden jurídico está compuesto de sistemas de normas, los que no tienen relaciones lógicas entre sí. Si esto es así, entonces el problema del hallazgo de una contradicción no tiene el efecto devastador de volver inaplicable el orden jurídico completo, sino que sólo perjudica al sistema en el que aparece.

Esta forma de identificar el modo de razonar de los juristas creo que es adecuado, y que una idea similar está reflejada en propuestas de Alchourrón y Bulygin ${ }^{8}$ de representar a las normas jurídicas como enunciados condicionales. Ellos definen a las normas jurídicas como enunciados que correlacionan casos con soluciones, estos enunciados pueden formularse como condicionales en cuyo antecedente se describe un caso y cuyo consecuente contiene una solución. El análisis que proponen consiste en trabajar a partir de un «universo del discurso», vale decir, a partir de la identificación de un conjunto de casos que tienen cierta propiedad en común y que son descriptos en el antecedente de alguna de las normas del sistema analizado; y a partir de un «universo de acciones» aquellas acciones genéricas que son calificadas deónticamente por tales normas. La caracterización deóntica de una conducta del Universo de Acciones constituye una

${ }^{7}$ Pazos, M. I. y Venier C. A., «La lógica deóntica como lógica del razonamiento jurídico», ponencia presentada en el Cologuio Interuniversitario sobre el tema «Lógica y Derecho», celebrado en la Sociedad Argentina de Análisis filosófico, Bs. As., 1994.

${ }^{8}$ Introducción a la metodología.... ob. cit. nota 1. 
solución. Las normas entonces enlazan la descripción de un caso con una solución, condicionando la solución al caso. Esto trae la consecuencia de que los sistemas analizados son subconjuntos del conjunto de todas las normas del Orden Jurídico, ya que para cada universo de casos y de soluciones sólo algunas normas del orden jurídico establecerán correlaciones entre sus elementos. En el análisis de Alchourrón y Bulygin es de estos sistemas de los que se predica consistencia o inconsistencia.

Estos autores creen que con cualquier conjunto de normas puede formarse un sistema, incluyendo el conjunto de todas las normas jurídicas de un ordenamiento positivo ${ }^{9}$, aunque construir un sistema tal no se hace de hecho jamás y hacerlo puede tener poco interés práctico. Estas ideas suponen que los juristas construyen los sistemas jurídicos antes de analizarlos, y que un sistema jurídico no existe hasta que el científico o el jurista lo crea. Los conceptos ordinarios de «sistema jurídico», en cambio, suponen que los sistemas ya existen, y que la tarea del jurista es meramente identificarlos. Creo, sin embargo, que la diferencia puede no ser genuina, sino meramente terminológica. Esto es así porque afirmar que a) los juristas reconocen sistemas jurídicos usando cierto método o concepto para formar un conjunto con ciertas normas y b) afirmar que los juristas construyen esos sistemas usando el mismo método, son dos opciones que conducen a la identificación de un sistema idéntico.

Sea que los juristas reconstruyan o identifiquen a los sistemas normativos a partir de un universo de casos y de acciones, se ve que la conexión entre las normas jurídicas y la forma lógica condicional es muy estrecha: Las normas jurídicas son enunciados que describen ciertas circunstancias a las que se subordina (condiciona) una solución (la caracterización deóntica de una conducta). Una norma afirma que si se dan las circunstancias de hecho (caso) entonces resultará obligatoria, prohibida o permitida alguna acción.

Vemos que la formulación de las normas jurídicas como condicionales parece adaptarse a las intuiciones de los juristas y a los conceptos ordinarios acerca de las normas jurídicas. Pero ¿qué sucedería si de hecho hubiera una norma positiva que calificara una conducta bajo cualquier circunstancia, es decir, una norma categórica? Tal norma tendría como condición de aplicación (antecedente) a cual-

${ }^{9}$ En este caso el universo de casos estará formado por todos los casos que puedan construirse a partir de descripciones de casos contenidas en las normas jurídicas. Similarmente el universo de acciones será el conjunto de todas aquellas que alguna norma del sistema caracterizara deónticamente. El universo del discurso consistirá en el conjunto de casos que tengan la propiedad común de poder obtenerse a partir del universo de casos determinado por todas las normas del sistema. 
quier circunstancia (caso). Si además tal norma fuese autocontradictoria, o si hubiese otra norma categórica que fuese contradictoria con ella, ¿qué consecuencias tendría esto?

En primer lugar, si consideramos que el orden jurídico en su totalidad es un sistema jurídico, como sostienen la mayoría de los autores, entonces simplemente sucederá que en el sistema total toda conducta descripta por alguna norma del sistema estará prohibida y permitida, y aquél resultará inaplicable.

$\mathrm{Si}$ en cambio consideramos que el orden jurídico es un conjunto de sistemas debemos considerar dos alternativas. Según la primera, un sistema se identifica por el conjunto de casos para los que se busca solución. Como una norma categórica es aplicable bajo cualquier circunstancia entonces también es aplicable para cualquier caso de cualquier conjunto, y en consecuencia pertenece a todos los sistemas volviéndolos inaplicables. Según la segunda alternativa un sistema jurídico se identifica tanto por un universo de casos como por un universo de acciones, de modo que la norma sólo pertenecerá a los sistemas que contengan entre las acciones que califica, a aquella conducta normada en forma contradictoria por la, o las, normas categóricas.

La última alternativa limita, en gran medida, el alcance de la dificultad. Creo, sin embargo, que el surgimiento mismo de ésta se debe a que el problema no ha sido bien planteado. En primer lugar, porque no se identificó el problema que preocupa a los juristas; y en segundo lugar porque se intenta resolver el problema de los juristas por el camino de solucionar problemas estrictamente lógicos: las razones que harían inaplicable la lógica al derecho. Éste es un problema previo, el de determinar cómo es o cómo debe ser la lógica adecuada (si alguna lo es) para dar cuenta de los problemas de los juristas tal como a éstos se les plantean. En particular para el análisis del problema de las contradicciones normativas.

La solución no puede consistir en demostrar que en grandes sectores del derecho no surgen contradicciones, porque esto es eludir la dificultad. Debe admitirse que los juristas reconocen un problema al que llaman «contradicción normativa» y determinarse si la lógica es un instrumento adecuado para identificarlo y, tal vez, resolverlo. Veamos la plausibilidad de una última salida en defensa de la lógica standard. Es probable que las consecuencias contraintuitivas del análisis lógico surjan de que se intenta resolver un problema dis tinto del que interesa a los juristas. Estos, al hablar de contradicciones, no se refieren a la contradicción de la lógica standard. Tal vez, identificado adecuadamente el problema se descubra que la lógica tradicional es útil para su análisis. 


\section{Redefinición del problema: la contradicción entre normas condicionales}

Notemos que la mayoría de las normas jurídicas son hipotéticas, y esto resulta claro si se piensa que es una característica típica de un sistema jurídico el que contenga enunciados generales, de la forma de los juicios categóricos universales aristotélicos. La forma standard de tales juicios en lógica de primer orden es la de un enunciado condicional universalmente cuantificado ${ }^{10}$. Es difícil imaginar ejemplos de normas jurídicas que no puedan ser formalizadas como condicionales (con antecedente contingente) y, si hubiera normas categóricas (lo cual es empíricamente posible) el problema sólo surgiría si fuesen contradictorias. De modo que el caso que tanto preocupa a los lógicos es un caso marginal con el que los juristas no se enfrentan y es probable que no haya una solución disponible entre los mecanismos que de hecho usan. Bien podría ser que el razonamiento jurídico, y la lógica presupuesta en éste, no estuviese preparada para dar una respuesta.

El lógico podría argumentar en este punto que el que no haya solución no significa que no haya problema, porque existe la posibilidad empírica de que se plantee. Podría responderse que no se requiere solución alguna en tanto no haya problema. No estoy segura de que esta respuesta sea del todo adecuada. Sin embargo, creo que hay una salida mejor. No es necesario responder a la pregunta de cómo podría resolverse el problema de las contradicciones si aparecieran normas categóricas contradictorias. Esto es así, porque el planteo del problema de las contradicciones normativas en el ámbito de las normas categóricas no es el adecuado. Las preocupaciones de los juristas surgen de hecho a menudo, pero se dan respecto de la aplicación de normas condicionales y casi siempre generales (es decir, formalizables como condicionales universalmente cuantificados). Es posible que haya un problema genuino con las normas categóricas posibles, pero el interés teórico en el tratamiento actual de las contradicciones normativas tiene el fin de ocuparse de aquello que interesa a los juristas.

Por otra parte creo que la respuesta al problema que se presenta respecto de las normas condicionales resolverá también cualquiera que pudiese originarse entre normas categóricas, aún cuando no suelan presentarse a los juristas casos de esa índole.

Que el problema a resolver no está bien identificado en el planteo lógico, es algo que se ve claramente si se observa que los pro-

${ }^{10}$ Ver por ejemplo Alchourrón, C., «Philosophical Foundations of Deontic Logic and the Logic of Defeasible Conditionals» (pág. 7). 
blemas interpretativos acerca de normas calificadas de «contradictorias» surgen a diario en la práctica jurídica y el estudio del derecho, de modo que no puede ser verdad que los casos de contradicciones son casos marginales, como son los que se refieren a contradicciones entre normas categóricas. Distintos autores señalaron ya que el problema standard de las contradicciones normativas se da más bien en casos en que son aplicables a una circunstancia fáctica varias normas condicionales que atribuyen a una conducta caracteres deónticos incompatibles, de modo que no pueden ser obedecidas ambas ${ }^{11}$. Por ejemplo dos normas que, respectivamente, permitieran y prohibieran una misma conducta bajo las mismas circunstancias.

La razón lógica para no llamar a estos casos «contradicciones» es que en el concepto tradicional de «contradicción» dos enunciados condicionales con consecuente inconsistente no son contradictorios, sino que, cuando tienen el mismo antecedente, son conjuntamente equivalentes a la negación de este último ${ }^{12}$. En caso de que el antecedente no fuese el mismo, no parece que pudiese detectarse un problema desde la lógica.

Pero es claro que el concepto de contradicción que usan los juristas no es el mismo de la lógica proposicional y cuanficacional tradicionales, de modo que debe redefinirse «contradicción» para dar cuenta de los problemas que enfrentan los juristas y que ellos mismos califican de «contradicciones normativas».

Ejemplos de contradicciones que los juristas reconocen son los siguientes. El caso más claro es el ya citado de dos normas con antecedente idéntico y consecuente contradictorio, o el caso (equivalente) de una norma con consecuente autocontradictorio. Como vimos, este tipo de normas trae como consecuencia el problema lógico de que se infiere la negación del antecedente, y así, de una contra-

${ }^{11}$ Ver por ejemplo Von Wright, G. H., Norma y Acción, ed. Tecnos, Madrid 1970 (Reimpresión 1979); Hilpinen, R. «Conflicts and Change in Normative Systems» (en Ake Frandberg y Mark Van Hoecke, Eds.), The Structure of Law, Iustus Forhg, Uppsala 1987; Alchourrón, C., «Conflictos de normas y revisión de sistemas normativos», «Condicionalidad y la representación de las normas jurídicas», ambos en Análisis lógico y Derecho, ob. cit. nota 1; Alchourrón, C., «Philosophical Foundations of Deontic Logic and the Logic or Defeasible Conditionals».

${ }^{12} \mathrm{Si}$ se considera la normalización de las normas como enunciados condicionales universalmente cuantificados, entonces dos normas de esta clase con consecuente contradictorio serán equivalentes a la negación del antecedente, universalmente cuantificado.

$$
[(\mathrm{x})(\mathrm{Px}>\mathrm{OS} \mathrm{x}) \&(\mathrm{x})(\mathrm{px}>-\mathrm{OS} \mathrm{x})] \equiv(\mathrm{x})-\mathrm{Px}
$$


dicción normativa se inferirían consecuencias ${ }^{13}$. Este problema no es identificado por los juristas como tal, dado que no efectúan de hecho esa inferencia. El segundo problema lógico, al que también nombramos, consiste en que las normas condicionales con consecuentes contradictorios implican lógicamente cualquier consecuencia. Esta dificultad puede limitarse como indicamos en el punto 3. Pero también es verdad para este problema lógico que los juristas no lo identifican como tal.

El caso más interesante que sí se plantea como problemático para la ciencia jurídica es aquél en que dos normas tienen consecuente inconsistente, pero diferentes condiciones de aplicación. El problema interpretativo surge cuando las condiciones de aplicación de ambas se producen, de hecho, simultáneamente. Este último caso muestra que dos normas pueden ser inconsistentes "vía determinados hechos"14.

Código: $\mathrm{Px}=\mathrm{x}$ es un menor que mató a alguien. $\mathrm{OSx}=$ Obligatorio que $\mathrm{x}$ sea sancionado.

(Ejemplo tomado de C. Alchourrón. Ver nota 14).

Demostración:

1) $(\mathrm{x})(\mathrm{Px}>\mathrm{OSx}) \&(\mathrm{x})(\mathrm{Px}>-\mathrm{OS} \mathrm{x})$.

2) $(\mathrm{Pa}>\mathrm{OSa}) \&(\mathrm{~Pa}>-\mathrm{Osa})$ de 1 (Instanciación Universal).

3) $\mathrm{Pa} \supset:($ Osa \&-Osa) de 2 equivalencia de lógica proposicional (LP).

4) -(OSa \& -OSa) principio de no-contrad. de LP y ley de Lógica Deóntica (LD)*

5) $-\mathrm{Pa}$ de 4 y 3 por Modus Tollens.

6) (x)-Pa Cuantificación universal.

1) (x) -Px

2) $-\mathrm{Pa}$ Instanciación Universal.

3) $-\mathrm{p}>[\mathrm{p}>(\mathrm{q} \&-\mathrm{g})] \quad$ Ley de LP.

4) $-\mathrm{Pa}>[\mathrm{Pa}>(\mathrm{OSa} \&-\mathrm{Osa})]$ Sustitución en 3.

5) $\mathrm{Pa}>(\mathrm{OSa} \&-\mathrm{OSa}) \quad$ De 4 y 2 Modus Ponens.

6) $(\mathrm{Pa}>\mathrm{OSa}) \&(\mathrm{~Pa}>-$ Osa De $5, \mathrm{LP}$

7) $(\mathrm{x})[(\mathrm{Px}>\mathrm{OSx}) \&(\mathrm{Px}>-\mathrm{Osx})]$ Cuantificación Universal.

8) $(\mathrm{x})(\mathrm{Px}>\mathrm{OSx}) \&(\mathrm{x})(\mathrm{Px}>-\mathrm{OSx})$ Distribución del Cuantificador.

*Alchourrón, C., "Lógica de normas y lógica de proposiciones normativas", en Alchourrón y Bulygin, Análisis lógico y Derecho, C.E.C., 1991, pág. 28.

Consideramos a este sistema como el sistema standard de L. D. Uno de sus teoremas es el siguiente:

T-11. (Pp v Vp)

que es equivalente a-(Op\&-Op).

${ }^{13}$ Alchourrón y Bulygin Introducción a Metodología... (op. cit. pág. 102).

${ }^{14} \mathrm{El}$ concepto de contradicción vía determinados hechos fue aclarado y analizado por $\mathrm{C}$. Alchourrón en "Conflictos de normas y revisión de sistemas normativos", ya citado. 
No voy a dar una definición precisa de contradicción normativa. Creo que la noción de contradicción "vía ciertos hechos" abarca a los demás tipos de inconsistencia. Consideraremos que un sistema normativo es inconsistente, según un nuevo sentido de la expresión, si es inconsistente vía algún conjunto de hechos (el caso límite es el del conjunto universal, vale decir, vía cualquier conjunto de hechos). Esto sucede cuando el sistema correlaciona con tales hechos dos soluciones incompatibles en el sentido de que no puede obedecerse a las prescripciones de ambas soluciones, por razones lógicas (vale decir, o bien se permite y prohíbe la misma conducta o bien se obliga y prohíbe la misma conducta, bajo las mismas circunstancias prohíbe).

Consideremos el caso citado por Alchourrón ${ }^{15}$ de un sistema del cual forman parte las normas siguientes:

a) Los que cometen un delito deben ser penados.

b) Los menores no deben ser penados.

Formalmente:

a) $\mathrm{p}>\mathrm{Oq}$

b) $\mathrm{r}>\mathrm{O}-\mathrm{q}$

El sistema es inconsistente vía la conjunción de hechos ( $\mathrm{p} \& \mathrm{r}$ ), pero no vía p ni vía r. Según la definición anterior, el sistema es inconsistente.

Hasta aquí vimos, en primer lugar, que el concepto de contradicción de la lógica tradicional no da cuenta del concepto que preocupa a los juristas $y$, en segundo lugar que tal concepto debe redefinirse en términos de inconsistencias entre soluciones normativas respecto de ciertos hechos o, lo que es equivalente, debe redefinirse la contradicción normativa como inconsistencia entre los consecuentes de normas condicionales aplicables a las mismas circunstancias (casos).

Esta forma de plantear el problema se acerca bastante, creo, a aquella en que lo plantean los juristas, de modo que estamos en el camino correcto.

\section{El problema de los lógicos, nuevamente}

Una vez identificado correctamente el concepto de contradicción o inconsistencia normativa, volvamos a analizar los efectos del argumento citado al principio contra el uso de la lógica formal en el análisis del derecho.

15 “Conflicto de normas y revisión...” ya citado, pág. 301. 
¿Cuáles serían los resultados (formales) del análisis de una contradicción como la que se da entre las normas a) yb)?

Para la lógica standard son válidos los dos razonamientos siguientes:

\section{Razonamiento A}

$$
\begin{aligned}
& \mathrm{p}>\mathrm{Oq} \\
& \mathrm{r}>\mathrm{O} \neg \mathrm{q}
\end{aligned}
$$

\section{Demostración}
1) $\mathrm{p}>\mathrm{Oq}$
2) $\mathrm{r}>\mathrm{O} \neg \mathrm{q}$
3) $(\mathrm{p} \cdot \mathrm{r})>(\mathrm{Oq} \cdot \mathrm{O} \neg \mathrm{q}) \mathrm{LP}$
4) $\neg(\mathrm{Oq} \cdot \mathrm{O} \neg \mathrm{q}) \mathrm{LP}$ y LD (ver nota 11$)$
5) $\neg(p \cdot q)$ de 3 y 4 Modus Tollens

De un par de normas, que como tales no describen, sino que prescriben, se infiere una proposición, i.e. un enunciado descriptivo que afirma que las condiciones de aplicación de la norma no se dan. Pero además esto demuestra que una contradicción normativa implica enunciados fácticos, los que pertenecerán también al sistema por ser consecuencias lógicas de enunciados del sistema.

\section{Razonamiento B}

$$
\begin{aligned}
& \mathrm{p}>\mathrm{Oq} \\
& \mathrm{r}>\mathrm{O}-\mathrm{q} \\
& (\mathrm{p} \cdot \mathrm{s})>\mathrm{s}
\end{aligned}
$$

Donde s representa cualquier enunciado.

\section{Demostración}

1) $\mathrm{p}>\mathrm{Oq}$ premisa

2) $\mathrm{r}>\mathrm{O} \neg$ q premisa

3) $\mathrm{O} \neg \mathrm{q}>\mathrm{P}$ q (Axioma Op > Pp de LD standard)

4) $\mathrm{r}>\mathrm{P} \neg \mathrm{q} \quad$ (De 2, 3 Sil.Hip)

5) $\mathrm{r}>\neg \mathrm{Oq} \quad$ (Interdf. $\mathrm{O}$ y $\mathrm{P})$

6) $(\mathrm{p} \cdot \mathrm{r})>(\mathrm{Oq} . \neg \mathrm{Oq}) \quad(1,5 \mathrm{LP})$ 
7) (Oq. $\neg$ Oq) $>$ s (Tesis de LP)

8) $(\mathrm{p} \cdot \mathrm{r})>\mathrm{s}(\mathrm{de} 6,7$ Sil. hip.)

Si de hecho ocurriera (p.r) el lógico podría inferir válidamente:

$(\mathrm{p} \cdot \mathrm{r})$

\section{S}

De este modo, en cualquier sistema normativo contradictorio, haciendo uso de la lógica standard, las circunstancias (casos) que sean antecedentes de normas contradictorias (en el sentido indicado de "contradicción") habilitarán a inferir cualquier enunciado. En la terminología de Alchourrón y Bulygin, el caso constituido por tales circunstancias estará correlacionado con cualquier solución (lo que sea una solución en el sistema puede estar restringido por las reglas acerca de la reconstrucción formal de un sistema jurídico, pero de cualquier modo el caso estará correlacionado con cualquier solución posible -cualquier fórmula bien formada que constituya una solución-).

Los resultados de A y B son completamente contraintuitivos, dado que extienden enormemente las consecuencias que un jurista, en su razonamiento usual, extraería a partir del reconocimiento de dos normas contradictorias. Para él, de la ocurrencia de las circunstancias $(p \cdot r)$, más las normas a) y b) del sistema, podría inferirse válidamente $\mathrm{Oq}$, $\mathrm{O} \neg \mathrm{q}$ y aún $(\mathrm{Oq} \cdot \mathrm{O} \neg \mathrm{q})$. En que todas éstas sean soluciones posibles consiste el problema teórico que preocupa a los juristas. Pero ellos no admitirían que puede inferirse $\mathrm{s}$, vale decir, cualquier otra solución; ni $\neg(\mathrm{p} \cdot \mathrm{q})$, es decir, el enunciado fáctico que afirma que las condiciones de aplicación de las normas contradictorias de hecho no se dan.

Esto prueba que hay algo malo con la lógica usada. No demuestra que la lógica deductiva formal sea inaplicable, sino que hay algo incorrecto en la medida en que, tal como está, permite validar inferencias intuitivamente inválidas.

El problema planteado no es el problema jurídico de las contradicciones normativas que identificamos en el parágrafo 1, sino una dificultad que enfrentan los lógicos: la lógica no reproduce adecuadamente lo que intenta representar, a saber, la forma de razonar usada por los juristas en el análisis del derecho. Si la lógica ha de emplearse en este análisis, entonces debe por una parte permitir identificar las contradicciones normativas que preocupan a los científicos del derecho (lo que vimos que ocurre si usamos una definición de "contradicción" adecuada), y por la otra debe permitir demostrar la validez de razonamientos jurídicos. Pero esta última 
función no se cumpliría adecuadamente si validara razonamientos que consideramos obviamente inválidos.

\section{La solución de C. Alchourrón ${ }^{2}$}

La idea de C. Alchourrón es que la razón por la cual aparecen contradicciones en un sistema es el uso en la lógica de las normas de los principios de la lógica tradicional. En particular, cualquier jurista que aceptara que las normas a) y b) pertenecen al sistema, estaría comprometido por los principios de la lógica standard a admitir que también pertenece la norma

$$
\text { c) }(\mathrm{p} \& \mathrm{r})>(\mathrm{Oq} \& \mathrm{O} \neg \mathrm{q})
$$

Pero, sostiene este autor, esto no sería aceptado por los juristas. Cualquiera de ellos y aún cualquier estudiante de derecho todavía poco conocedor del derecho argentino contestaría que la respuesta que da el derecho para el caso en que ambos antecedentes concurrieran, es decir, para el caso en que un menor delinquiera, es la dispuesta en la norma b) (un menor -aunque cometa un delito- no debe ser penado).

Alchourrón da fundamentos para rechazar de la lógica deóntica dos principios de la lógica tradicional. No puede valer el principio de refuerzo del antecedente, porque la concurrencia de nuevas circunstancias podría hacer que no se infiriera el consecuente de una norma del sistema. Del mismo modo debe ser inválida la regla del modus ponnens, dado que el que se dé en la realidad el antecedente de una norma no garantiza que deba inferirse el consecuente, vale decir, que sea aplicable la solución dispuesta por la norma en cuestión. Si alguien comete un delito, entonces debe ser penado, pero no puede inferirse sencillamente que alguien deba ser penado del hecho de que delinca, porque podría suceder que el que lo hizo sea un menor, caso en el que no debería aplicarse sanción.

Así propone Alchourrón que un sistema jurídico sea formalizado como un conjunto de normas condicionales ligadas por una conectiva más débil que el condicional material. Los enunciados en cuestión responderán a una lógica no-monotónica (no serán válidos los dos principios citados). La lógica de los condicionales derrotables.

En el sistema que analizamos, las dos normas citadas pueden formalizarse, usando el símbolo ">" para la nueva conectiva, de la siguiente manera:

a') $\mathrm{p}>\mathrm{Oq}$

b') $\mathrm{r}>\mathrm{O} \neg \mathrm{q}$ 
El último elemento que introduce es el concepto de "ordenación”. Las normas del sistema deben ser ordenadas jerárquicamente, i. e. establecer algún tipo de prioridad entre ellas. La ordenación es parte del contenido del sistema y es necesaria para la determinación de sus consecuencias, vale decir de las soluciones jurídicas.

El conocimiento de las normas que integren el sistema, más la información adicional acerca de la ordenación entre éstas, hará posible inferir las respuestas para las distintas circunstancias posibles $(\mathrm{p} \cdot \mathrm{r}),(\neg \mathrm{p} \cdot \mathrm{r}) \mathrm{y}(\mathrm{p} \cdot \neg \mathrm{r})$. Tales respuestas serán, para nuestro caso, las siguientes:

c) Si un menor comete un delito no debe ser penado.

d) Si alguien que no es menor comete un delito, debe ser penado.

e) Si un menor no comete un delito, no debe ser penado.

Estos enunciados constituyen las soluciones que el sistema proporciona y pueden formalizarse del siguiente modo, donde el esquema $\mathrm{O}(\alpha / \beta)$ indica la obligación de realizar la conducta $\alpha$ bajo las condiciones $\beta$ (siendo $\alpha$ cualquier conducta y $\beta$ cualquier condición o conjunto de condiciones).
c') $\mathrm{O}(-\mathrm{q} / \mathrm{p} \cdot \mathrm{r})$
d') $\mathrm{O}(\mathrm{q} / \mathrm{-p} \cdot \mathrm{r})$
e') $\mathrm{O}(-\mathrm{q} / \mathrm{p} \cdot-\mathrm{r})$

También surgen del sistema las obligaciones:
f) $\mathrm{O}(-\mathrm{q} / \mathrm{p})$
g) $\mathrm{O}(\mathrm{q} / \mathrm{r})$

Todas son obligaciones derrotables.

Las soluciones a que lleva esta forma de interpretar las normas originales se adapta a las intuiciones de las que partimos. La explicación de por qué las soluciones implicadas en el sistema son c), d) y e), y no por ej. "Si un menor comete un delito debe ser penado" (que podría considerarse derivable de la norma a'), es que b' en algún sentido tiene prioridad sobre a', de modo que, en caso de coincidencia en sus condiciones de aplicación, la solución correcta será la dispuesta por b'. Esta prioridad está presupuesta en el derecho y forma parte de su contenido. La expresión de la norma condicional derrotable según la cual el que comete un delito debe ser penado, presupone que no se trata de un menor. 


\section{El método para determinar las obligaciones (derrotables) según un sistema normativo}

Para conocer las obligaciones que surgen de un sistema normativo A, según el método propuesto por Alchourrón, deben seguirse los siguientes pasos:

I. Para cada circunstancia fáctica (nombrada en el antecedente de alguna norma del sistema) o combinación posible de circunstancias t (en adelante "caso" se busca en el sistema el mínimo subconjunto de normas de A que, si se formalizara a las normas como condicionales materiales, originaría soluciones contradictorias (para ese caso). Se llama a tal subconjunto "subconjunto crítico" para t en A. Puede no haber subconjunto crítico para todo caso t.

II. Se determina una ordenación de las normas del sistema, de a pares. No se requiere que todas las normas se relacionen entre sí por esta relación de ordenación, ni que todas tengan esta relación con alguna otra norma.

III. Dentro de cada subconjunto crítico (para algún caso t en A) se elimina la norma de jerarquía inferior, i. e. aquella que no sea prioritaria respecto de ninguna otra del subconjunto. Esta operación no requiere que la norma eliminada tenga una superior dentro del subconjunto crítico, o dentro del sistema.

IV. Se determinan los llamados "conjuntos sanos" respecto de cada caso t. Un conjunto sano para $t$ es el constituido por todas las normas del sistema que no hayan sido eliminadas del conjunto crítico respecto de $\mathrm{t}$.

V. Finalmente puede determinarse el conjunto de obligaciones derrotables del sistema. Cada caso t estará correlacionado en A con la(s) solucione(s) que surjan del subconjunto de normas sano para t.

Siguiendo estas instrucciones para el análisis del sistema presentado antes, obtendríamos las soluciones c'a g indicadas antes.

Una vez determinadas las obligaciones derrotables del sistema puede indicarse cuáles son las obligaciones inderrotables: aquellas que no podrían ser derrotadas en el sistema por la conjunción de nuevos hechos. En nuestro caso, c'), d') y e') constituyen obligaciones inderrotables en A. Para ellas valen los principios de la lógica standard. 


\section{Normas de igual jerarquía}

Volvamos al ejemplo usado en el punto 1, considerando el sistema normativo S que consta de las dos normas siguientes.

a) Obligatorio detenerse ante los semáforos en rojo.

b) Prohibido detenerse en zona militar.

Las que podrían formalizarse en términos de condicionales derrotables como:
a') $\mathrm{p}>\mathrm{Oq}$
b') $\mathrm{r}>\mathrm{O}-\mathrm{q}$

Este sistema origina problemas únicamente para el caso $\mathrm{p} \cdot \mathrm{r}$, vale decir, si se emplazara un semáforo en zona militar, caso para el cual a) y b) disponen que es obligatorio detenerse y no detenerse, respectivamente.

Para el caso $\mathrm{p} \cdot \mathrm{r}$, encontramos en $\mathrm{S}$ el subconjunto crítico

$$
\{\mathrm{p}>\mathrm{Oq}, \mathrm{r}>\mathrm{O}-\mathrm{q}\}
$$

El paso siguiente en la determinación de las obligaciones dispuestas por S, consiste en fijar la ordenación o jerarquía entre las normas de S. Esto es, debemos determinar si el sistema dispone que los automovilistas deben detenerse ante los semáforos en rojo aún en zona militar [prioridad de a) sobre b)], o si en zona militar es obligatorio no detenerse aún cuando se pasen semáforos en rojo [prioridad de b) sobre a)].

Pero aquí no parece claro cuál norma prevalece. Es muy probable que éste, como muchos otros casos del derecho positivo, no haya sido previsto cuando se redactaron las normas y que no pueda establecerse una relación de prioridad ni explícita ni implícita entre las normas que originan el conflicto. Si en el sistema no hay una relación de prioridad entre las normas que pertenecen a un mismo subconjunto crítico, entonces ninguna de ellas tendrá a otra como superior, y por lo tanto ambas serán de jerarquía inferior en el sentido indicado en el paso III.

La consecuencia de que ambas normas sean las de menor jerarquía del subconjunto es que ambas deben ser eliminadas de éste, de modo que el subconjunto sano respecto de ese caso no contendrá a ninguna de ellas. El caso no tendrá solución en el sistema. La justificación que da Alchourrón de esta medida consiste en afirmar que debe eliminarse del sistema lo mínimo indispensable 
para evitar las contradicciones. Por una parte, el argumento es erróneo dado que lo mínimo indispensable es eliminar cualquiera de las dos normas, no ambas. Eliminar lo mínimo implica eliminar una norma, antes que dos. Pero creo que el punto importante a tratar consiste en que debe justificarse la operación de eliminar normas del sistema, cualquiera sea el método que se use para decidir cuál suprimir. Veamos si esta justificación es posible.

\section{La reconstrucción lógica del derecho}

La aplicación de la lógica al análisis del derecho positivo, tiene por fin, como dijimos, el de ser un instrumento útil para el planteo y tratamiento de los problemas que de hecho se plantean a los juristas. Debe representar, por una parte, el tipo de relaciones que ellos ven entre las normas y, por la otra, reflejar las formas de razonamientos que consideran válidos. La formalización del sistema debe representar al sistema jurídico que los científicos del derecho reconocen como tal, y las reglas de la lógica deben permitir realizar sobre el derecho formalizado el tipo de inferencias o de operaciones lógicas que de hecho aquellos realicen o reconozcan como admisibles.

Si esto es así, entonces la formalización de un conjunto de normas positivas debe ser tal que, si se interpretan las fórmulas asignando un significado a las variables, resulte el conjunto de enunciados que (o cuyos significados) conformen el derecho positivo sobre el que se trabaja.

Esto implica que la reconstrucción lógica del derecho no es una tarea de creación de derecho, sino de representación de aquello que el concepto de derecho usado reconoce como tal. De hecho sucede que los juristas consideran que las normas jurídicas forman sistemas. La función de la lógica es representar este hecho, y no "agregarlo" a algún conjunto de enunciados no sistemático.

Se infiere que la lógica debe dar cuenta de los problemas que los juristas detectan en el derecho, pero no modificarlo. Ahora bien, creo que la lógica, aplicada al derecho debe ser capaz de reproducir dos intuiciones básicas. La primera se relaciona con el contenido del derecho: hay normas jurídicas contradictorias; la segunda está ligada a la clase de inferencias jurídicamente admisibles: de una contradicción normativa no puede inferirse cualquier solución para el caso al que se aplica, sino cualquiera de las soluciones contradictorias, o ambas, pero no otra. En particular no puede afirmarse que de todo par de normas contradictorias no se infiera ninguna de ellas, porque esto o sería autocontradictorio, o implicaría que las normas no estaban en el sistema. Esta última alternativa es 
equivalente a decir que los sistemas jurídicos no son contradictorios, lo cual se opone a la primera intuición de la que queremos dar cuenta: la existencia de contradicciones.

Ahora evaluemos la propuesta de C. Alchourrón.

En primer lugar, la interpretación de las normas formuladas como condicionales derrotables, refleja adecuadamente el hecho de que en muchas ocasiones existe una jerarquía explícita o implícita (presupuesta) entre las normas de modo que no surgen contradicciones para estos casos.

En segundo lugar, la lógica de los condicionales derrotables, al evitar que en estos casos surjan contradicciones, impide que se infiera para ellos cualquier solución. Sólo habrá una.

Pero estas son ventajas en el tratamiento de casos en que de hecho no hay contradicciones, justamente porque hay una jerarquía entre las normas aparentemente contradictorias. Veamos que ocurre cuando un sistema es inconsistente.

Sucede que cuando dos normas contradictorias tienen la misma jerarquía, el sistema es contradictorio y éste es problema que la lógica debe ser capaz de reconocer sin que se infieran las consecuencias contraintuitivas ya aludidas. Alchourrón resuelve estos casos eliminando del sistema a ambas normas, por ser ambas de jerarquía inferior (aún cuando no haya una superior). Esto supone:

a) o bien que los sistemas normativos nunca son inconsistentes, porque las normas contradictorias no pertenecen al sistema, o bien

b) que la operación de formalizarlos no intenta reflejar el derecho, sino modificarlo, eliminando las contradicciones.

Como ambas alternativas fueron expresamente rechazadas, sólo queda reconocer que la propuesta es inaceptable.

\section{Un intento de salvar a los condicionales derrotables}

No es necesaria una modificación importante de la interpretación de los condicionales derrotables, para evitar la indeseable consecuencia de que toda contradicción quede eliminada del sistema, preservando a la vez el tratamiento adecuado de los casos de jerarquía entre normas sólo aparentemente contradictorias. Es suficiente un pequeño cambio en el paso III del método para la determinación de las obligaciones del sistema.

Una vez identificados los subconjuntos críticos respecto de cada caso, se eliminará de cada uno de ellos la norma inferior, pero sólo en caso de que haya en el mismo subconjunto una que sea priorita- 
ria. Esto da cuenta del hecho de que cuando hay normas de diferente jerarquía es aplicable la superior, pero cuando no hay una relación jerárquica tal, hay en el sistema normas contradictorias.

Esta alternativa, claramente, preserva las contradicciones en el sistema. S determinará las obligaciones condicionales inderrotables $\mathrm{O}(\mathrm{q} / / \mathrm{p} \cdot \mathrm{r})$ y $\mathrm{O}(\neg \mathrm{q} / / \mathrm{p} \cdot \mathrm{r})^{16}$, que son contradictorias. Para ellas es aplicable la lógica standard y en ella de una contradicción cualquier consecuencia es derivable. Éste es el tipo de razonamiento inválido que debe evitarse, reemplazándolo por uno que permita inferir conclusiones jurídicamente aceptables. Se requiere una lógica con un concepto de deducibilidad más riguroso.

\section{Conclusión}

La conclusión es que antes de preocuparse por resolver el problema de los juristas de cómo hacer frente a las contradicciones normativas (el problema de que arrojan un par de soluciones incompatibles), debe contarse con una lógica que permita reconocer este problema en lugar de reemplazarlo por uno que no existe: el de que una contradicción normativa implica cualquier solución.

El problema de los juristas no puede resolverse desde la lógica.

El problema de los lógicos todavía no ha sido resuelto.

${ }^{16}$ Donde la barra doble (//) indica la inderrotabilidad de la obligación condicional. 